Pacific Journal of Mathematics

ALGEBRAS WHICH SATISFY A SECOND ORDER LINEA 


\title{
ALGEBRAS WHICH SATISFY A SECOND ORDER LINEAR PARTIAL DIFFERENTIAL EQUATION
}

\author{
H. S. Bear and G. N. Hile
}

Let $A$ be an algebra of complex valued functions satisfying a second order linear partial differential equation in a plane domain. If the equation is hyperbolic or parabolic, the functions of $A$ are locally functions of only one variable. If the equation is elliptic, there exists a unique complex function $\lambda$ such that $f_{x}=\lambda f_{y}$ for each $f$ in $A$, and after a change of variables each function in $A$ is analytic. If an algebra of functions satisfies the maximum principle, and one nonconstant function and its square satisfy an elliptic equation, then every function in the algebra satisfies this equation.

1. Introduction. In this paper we study algebras of complex valued functions defined on a plane domain, which satisfy some linear second order partial differenital equation

$$
L w=a w_{x x}+2 b w_{x y}+c w_{y y}+d w_{x}+e w_{y}=0,
$$

with real coefficients. We start with an example which turns out to be typical of the significant cases.

Let $L$ be a self-adjoint elliptic operator:

$$
L w=\frac{\partial}{\partial x}\left(a w_{x}+b w_{y}\right)+\frac{\partial}{\partial y}\left(b w_{x}+c w_{y}\right)
$$

where $a, b, c$ are $C^{2}$ real functions on a simply connected domain, satisfying the normalizing condition $a c-b^{2}=1$. For each $C^{2}$ function $u$ satisfying $L u=0$, we define (up to an additive constant) a conjugate function $v$ by

$$
v(x, y)=\int^{(x, y)}-\left(b u_{x}+c u_{y}\right) d x+\left(a u_{x}+b u_{y}\right) d y .
$$

It is easy to check the following facts: $L v=0$; the conjugate of $v$ is $-u$; the set of functions $u+i v$ is an algebra; $(u+i v)^{-1}$ is in the algebra if $u+i v \neq 0$.

The functions $u+i v$ turn out to be analytic after the appropriate change of variables. Moreover, the example illustrates the only way 
that the functions of an algebra can satisfy a linear second order elliptic partial differential equation.

Suppose $A$ is a function algebra (a Banach algebra of complex continuous functions, with the sup norm) on the unit circle $\Gamma=\{z:|z|=$ 1\}. If $\operatorname{Re} A=\operatorname{Re} A_{0}$, where $A_{0}$ is the disc algebra restricted to the circle, then $[4,5] A=A_{0} \circ \Phi$ for some homeomorphism $\Phi$ of $\Gamma$ onto $\Gamma$. We obtain a similar result for algebras defined on a domain rather than on its boundary. Specifically, if $A$ is an algebra of functions on a domain and $\operatorname{Re} A$ consists of harmonic functions, then $A$ or $\bar{A}$ consists of analytic functions.

We also obtain a simple geometric characterization of functions which are analytic functions of a homeomorphism (i.e., interior mappings in the sense of Stoilow). Let $u, v$ be sufficiently smooth real functions on a domain $G$. Then $u+i v$ or $u-i v$ is analytic on $G$ if and only if $\nabla u \cdot \nabla v=0$ and $|\nabla u|=|\nabla v|$ on $G$. This result generalizes as follows. We define a family of inner products " $*$ ", each with its norm " \|\|$"$. For each such inner product $*$, the equations $\nabla u * \nabla v=0$ and $\|\nabla u\|=\|\nabla v\|$ characterize those functions $u+i v$ which are analytic after a change of variables determined by $*$. The equations imply that $\nabla u$ and $\nabla v$ are nonparallel wherever they are nonzero. The converse is essentially true. In particular, if $\nabla u$ and $\nabla v$ never vanish and are never parallel on a domain, then $u+i v$ is analytic after an appropriate change of variables.

In the final section we apply our results to algebras which satisfy a maximum principle on $G$, and obtain two extensions of results of Rudin [7] for such function algebras.

2. The parabolic and hyperbolic cases. In this section we consider algebras of complex $C^{2}$ functions which satisfy (1), where $L$ is parabolic or hyperbolic. We show that no such algebra can separate points, and in fact must consist essentially of functions of one variable.

We assume that the coefficients of $L$ are real $C^{2}$ functions on a domain $G$ in the $(x, y)$-plane, and that $a, b, c$ do not vanish simultaneously. A solution of (1) is a real or complex $C^{2}$ function which satisfies (1) identically on $G$.

An "algebra of functions" on $G$ will always be assumed to contain at least one non-constant function.

A "change of variables" means a one-to-one transformation $(x, y) \rightarrow(\xi, \eta)$ where $\xi=\xi(x, y), \eta=\eta(x, y)$ are $C^{2}$ functions and the Jacobian $\xi_{x} \eta_{y}-\xi_{y} \eta_{x}$ does not vanish. It follows that the inverse functions $x=x(\xi, \eta), y=y(\xi, \eta)$ are also $C^{2}$. The equation (1) transforms into the following equivalent equation in the $(\xi, \eta)$ variables: 


$$
L^{\prime} w=a^{\prime} w_{\xi \xi}+2 b^{\prime} w_{\xi \eta}+c^{\prime} w_{\eta \eta}+d^{\prime} w_{\xi}+e^{\prime} w_{\eta}=0,
$$

where

$$
\begin{aligned}
& a^{\prime}=a \xi_{x}^{2}+2 b \xi_{x} \xi_{y}+c \xi_{y}^{2}, \\
& b^{\prime}=a \xi_{x} \eta_{x}+b \xi_{x} \eta_{y}+b \xi_{y} \eta_{x}+c \xi_{y} \eta_{y}, \\
& c^{\prime}=a \eta_{x}^{2}+2 b \eta_{x} \eta_{y}+c \eta_{y}^{2}, \\
& d^{\prime}=L \xi \\
& e^{\prime}=L \eta .
\end{aligned}
$$

Clearly $a^{\prime}, b^{\prime}, c^{\prime}$ are $C^{1}$ functions, and $d^{\prime}, e^{\prime}$ are continuous.

If (1) is a parabolic equation (i.e., $a c-b^{2}=0$ in $G$ ), then for each point of $G$ there is a neighborhood $U$, and a change of variables on $U$ onto $U^{\prime}$ so that the equation takes the form

$$
L^{\prime} w=w_{\xi \xi}+d^{\prime} w_{\xi}+e^{\prime} w_{\eta}=0
$$

on $U^{\prime}[6$, p. 63].

If (1) is hyperbolic $\left(a c-b^{2}<0\right.$ in $\left.G\right)$, then each point of $G$ has a neighborhood $U$ and a change of variables on $U$ onto $U^{\prime}$ so that the equation takes the form

$$
L^{\prime \prime} w=w_{\xi \eta}+d^{\prime} w_{\xi}+e^{\prime} w_{\eta}=0
$$

on $U^{\prime}[6$, p. 58].

We first look at algebras which satisfy a parabolic or hyperbolic equation in canonical form. To this end define the operators $M$ and $N$ as follows ( $d$ and $e$ are continuous functions):

$$
\begin{aligned}
& M w=w_{x x}+d w_{x}+e w_{y}, \\
& N w=w_{x y}+d w_{x}+e w_{y} .
\end{aligned}
$$

THEOREM 1. Let $A$ be an algebra of complex $C^{2}$ functions which satisfy the parabolic equation $M f=0$. Then each $f$ in $A$ is a function of $y$ only.

Proof. Let $f=u+i v \in A$. Since $f^{2} \in A, u^{2}-v^{2}$ and $u v$ also satisfy $M w=0$. Setting $M(u v)=0$ and using $M u=M v=0$ we conclude that $u_{x} v_{x}=0$. Similarly, $M\left(u^{2}-v^{2}\right)=0$ leads to $u_{x}^{2}=v_{x}^{2}$. Hence $u_{x}=v_{x}=0$, and $f$ is a function of $y$. 
COROllary. If $A$ is an algebra of complex $C^{2}$ functions which satisfy the parabolic equation (1) on $G$, then each function of $A$ is locally a function of the same single variable after a change of coordinates.

Now we turn to the hyperbolic case.

LEMMA 2. Let $A$ be an algebra of complex $C^{2}$ functions on $G$ such that $N f=0$ for all $f \in A$. If $A$ contains some function $f$ such that $f_{x}$ does not vanish on $G$, then every function in $A$ is a function of $x$ only. Similarly, if $A$ contains a function $g$ such that $g_{y}$ does not vanish on $G$, then every function in $A$ is a function of $y$ only.

Proof. Let $f=u+i v \in A$. Using the equations $N u=N v=$ $N(u v)=N\left(u^{2}-v^{2}\right)=0$ we obtain

$$
\begin{aligned}
& u_{x} v_{y}+u_{y} v_{x}=0 \\
& u_{x} u_{y}-v_{y} v_{x}=0
\end{aligned}
$$

Considered as equations in $u_{x}, v_{x}$, the determinant is $-\left(u_{y}^{2}+v_{y}^{2}\right)$. Hence $\left(u_{x}, v_{x}\right) \neq(0,0)$ implies $\left(u_{y}, v_{y}\right)=(0,0)$. Similarly, $\left(u_{y}, v_{y}\right) \neq(0,0)$ implies $\left(u_{x}, v_{x}\right)=(0,0)$, and $f_{x}, f_{y}$ cannot both be nonzero at the same point. If $f_{x}$ does not vanish in $G$, then $f_{y} \equiv 0$ in $G$, and $f$ is a function of $x$. Similarly, if $g_{y}$ does not vanish on $G$, then $g$ is a function of $y$ only. If $f_{x}$ does not vanish on $G$, then $g_{y} \equiv 0$ for all $g \in A$. Otherwise, if $g_{y}\left(x_{0}, y_{0}\right) \neq 0$, we let $h=f+g$, and get the contradiction $h_{x}\left(x_{0}, y_{0}\right)=f_{x}\left(x_{0}, y_{0}\right) \neq 0$, and $h_{y}\left(x_{0}, y_{0}\right)=g_{y}\left(x_{0}, y_{0}\right) \neq 0$.

THEOREM 3. Let $A$ be an algebra of complex $C^{2}$ functions on a domain $G$, such that $L f=0$ for all $f \in A$, where $L$ is hyperbolic. If $f_{x}\left(x_{0}, y_{0}\right) \neq 0$ or $f_{y}\left(x_{0}, y_{0}\right) \neq 0$ for some $f \in A$ and some $\left(x_{0}, y_{0}\right) \in G$, then there is a neighborhood $U$ of $\left(x_{0}, y_{0}\right)$ and a change of variables $(x, y) \rightarrow(\xi, \eta)$ on $U$ onto $U^{\prime}$ such that every function in $A$ is a function of $\xi$ on $U^{\prime}$, or every function in $A$ is a function of $\eta$ on $U^{\prime}$.

Proof. We make a local change of variables so that $L f=0$ becomes (7) on $U^{\prime}$. Since $f_{x}=f_{\xi} \xi_{x}+f_{\eta} \eta_{x}, f_{y}=f_{\xi} \xi_{y}+f_{\eta} \eta_{y}$, either $f_{\xi}$ or $f_{\eta}$ is nonzero in a sufficiently small neighborhood of $\left(\xi\left(x_{0}, y_{0}\right), \eta\left(x_{0}, y_{0}\right)\right)$. The result then follows from Lemma 2.

The following example shows that when the functions of an algebra satisfy a hyperbolic equation, these functions need not be globally functions of the same variable. Let $S=\{(x, y):|x|<1,|y|<1\}$ and let $G$ be $S$ with the closed first quadrant removed. Let $f(x, y)=y^{3}$ in the 
second quadrant, $f(x, y)=0$ in the third quadrant, and $f(x, y)=x^{3}$ in the fourth quadrant. All polynomials in $f$ satisfy the hyperbolic equation $w_{x y}=0$.

3. Elliptic case with Laplacian principal part. We consider now the following elliptic equation:

$$
L_{0} w=w_{x x}+w_{y y}+d w_{x}+e w_{y}=0 \text {, }
$$

where $d$ and $e$ are continuous real functions on a domain $G$. We show that if $A$ is an algebra of complex $C^{2}$ functions on $G$ which satisfy (11), then $A$ or $\bar{A}$ consists of analytic functions, and $d=e=0$.

The following theorem gives a very simple and appealing geometric description of how the gradients of real functions $u$ and $v$ must behave in order for $u+i v$ to be a conformal mapping. We say that $u$ and $v$ are conjugate harmonic functions in a domain if either $u+i v$ or $u-i v$ is analytic in that domain.

THEOREM 4. If $u, v$ are $C^{2}$ functions on a domain $G$, then $u$ and $v$ are conjugate harmonic functions in $G$ if and only if

$$
\nabla u \cdot \nabla v=0, \quad|\nabla u|=|\nabla v|
$$

hold identically in $G$.

Note. The result above was stated by Dzyadyk [3] for $C^{1}$ functions but there appears to be a gap in the proof at the following point. Let $\phi$ be continuous on a domain $G$, zero on a closed set $Z$ contained in $G$, and analytic on each component of $G-Z$. Then (?) $G-Z$ has only one component and $\phi$ is analytic in $G$. We do not know a proof of this statement. ${ }^{1}$ However for our purposes we only require the result for $C^{2}$ functions, and for this case we furnish the elementary proof below.

Proof. If either $f$ or $\bar{f}$ is analytic, then (12) follows from the Cauchy-Riemann equations. We assume therefore that (12) holds. If $f=u+i v$, then (12) is equivalent to $f_{x}^{2}+f_{y}^{2}=0$. Hence $f_{x}=0$ if and only if $f_{y}=0$, and $f_{x}= \pm i f_{y}$. Let $Z=\left\{(x, y): u_{x}=u_{y}=v_{x}=v_{y}=0\right\}$. Then $G-Z=Z^{c}$ is open, and $Z^{0} \cup Z^{c}$ is dense in $G$. Clearly $u$ and $v$ are harmonic on $Z^{0}$. Since $f_{x}= \pm i f_{y}$ with one sign holding on each component of $Z^{c}, f$ or $\bar{f}$ is analytic on each component of $Z^{c}$. Hence $u$

${ }^{1}$ We are indebted to Walter Rudin for pointing out that this statement is a Theorem of Radó (see, e.g. [8, Theorem 12.13]). 
and $v$ are harmonic on $Z^{0} \cup Z^{c}$, and by the continuity of $u_{x x}+u_{y y}$, $v_{x x}+v_{y y}, u$ and $v$ are harmonic on $G$. The functions $g=u_{x}-i u_{y}$ and $h=v_{x}-i v_{y}$ are analytic in $G$, and hence have isolated zeros (unless $u$ and $v$ are constant). Therefore $Z$ consists of isolated points, $Z^{0}$ is empty, and $Z^{c}$ is connected and dense. Hence $f$ or $\bar{f}$ is analytic on all of $Z^{c}$. Since $f$ is continuous on $G, f$ or $\bar{f}$ is analytic on $G$.

DEFINITION. We say $u$ and $v$ are square-conjugates for $L$ if and only if $u$ or $v$ is nonconstant, and $L u=L v=L(u v)=L\left(u^{2}-v^{2}\right)=0$. The last condition is of course equivalent to $L(f)=L\left(f^{2}\right)=0$, where $f=u+i v$.

THEOREM 5. If $u, v$ are $C^{2}$ functions in $G$ which are squareconjugate for $L_{0}$, then $u$ and $v$ are conjugate harmonic functions in $G$; moreover, $d=e=0$, and $L_{0}$ is the Laplacian.

Proof. We calculate as follows:

$$
\begin{aligned}
& L_{0}(u v)=u L_{0}(v)+v L_{0}(u)+2 \nabla u \cdot \nabla v \\
& L_{0}\left(u^{2}-v^{2}\right)=2 u L_{0}(u)-2 v L_{0}(v)+2\left(|\nabla u|^{2}-|\nabla v|^{2}\right) .
\end{aligned}
$$

If $u$ and $v$ are square-conjugates, then $\nabla u \cdot \nabla v=0$, and $|\nabla u|=$ $|\nabla v|$, and $u, v$ are harmonic conjugates by Theorem 4 .

Since $u$ and $v$ are harmonic and $L_{0} u=L_{0} v=0$, we also have

$$
\begin{aligned}
& d u_{x}+e u_{y}=0, \\
& d v_{x}+e v_{y}=0 .
\end{aligned}
$$

Let $f=u \pm i v$ (whichever is analytic in $G$ ). The determinant of the system (13) is $\pm\left|f^{\prime}\right|^{2}$. Since $u$ or $v$ is nonconstant, the determinant vanishes at most at isolated points of $G$, and off this set $d=e=0$. By continuity, $d=e=0$ on $G$.

The following theorem says in particular that function algebras whose real parts are harmonic functions consist of analytic functions, or consist of conjugates of analytic functions.

THEOREM 6. If $A$ is an algebra of complex $C^{2}$ functions on $G$, and $L_{0} f=0$ for all $f \in A$, then $A$ or $\bar{A}$ consists of analytic functions, and $L_{0}$ is the Laplacian.

Proof. Let $f=u+i v$ be a nonconstant function in $A$. Then $u$ and $v$ are square-conjugates for $L_{0}, f$ or $\bar{f}$ is analytic, and $d=e=0$. We 
need only show that $A$ cannot contain both a nonconstant analytic function and the conjugate of a nonconstant analytic function. Suppose on the contrary that $g, \bar{h} \in A$, with $g$ and $h$ analytic and nonconstant. Then $g \bar{h}$ or $\bar{g} h$ is analytic. If, for example, $g \bar{h}$ is analytic, then $\bar{h}$ is analytic except on the set of isolated points where $g$ is zero. Since $h$ is continuous, $h$ and $\bar{h}$ are both analytic, which is a contradiction.

4. The general elliptic case. We now consider an elliptic equation of the form

$$
L w=a w_{x x}+2 b w_{x y}+c w_{y y}+d w_{x}+e w_{y}=0 .
$$

We will make standard assumptions on the coefficients in terms of the following definitions.

Definition. A function $f$ is Hölder continuous in $G$ if for every compact subset $K$ of $G$ there are positive constants $c, \alpha$, with $0<\alpha \leqq 1$, such that $\left|f\left(z_{1}\right)-f\left(z_{2}\right)\right| \leqq c\left|z_{1}-z_{2}\right|^{\alpha}$ for all $z_{1}, z_{2} \in K$. A function $f$ is in the class $H_{m}(G)$ if $f$ and its partial derivatives up to order $m$ are Hölder continuous in $G$.

Definition. We will say that the operator $L$ of (14) is a regular elliptic operator in $G$ if $a, b, c \in H_{1}(G), d, e$ are continuous on $G$, and the two normalizing conditions hold: $a c-b^{2}=1, a>0$.

LEMMA 7. If $u, v$ satisfy (14), then $L(u v)=0$ if and only if

$$
\nabla u * \nabla v=0
$$

and $L\left(u^{2}-v^{2}\right)=0$ if and only if

$$
\|\nabla u\|=\|\nabla v\|
$$

where

$$
\begin{aligned}
\nabla u * \nabla u & \equiv a u_{x} v_{x}+b u_{x} v_{y}+b u_{y} v_{x}+c u_{y} v_{y} \\
\|\nabla u\|^{2} & \equiv \nabla u * \nabla u=a u_{x}^{2}+2 b u_{x} u_{y}+c u_{y}^{2}
\end{aligned}
$$

If $f=u+i v$, then (15) and (16) together are equivalent to the complex form

$$
a f_{x}^{2}+2 b f_{x} f_{y}+c f_{y}^{2}=0 .
$$


Proof. The results are easily verified by computation, and do not depend on the ellipticity of $L$.

We now let $L$ be the regular elliptic operator of (14), and consider the following Beltrami system associated with $L$ :

$$
\begin{aligned}
& \eta_{x}=b \xi_{x}+c \xi_{y} \\
& \eta_{y}=-a \xi_{x}-b \xi_{y} .
\end{aligned}
$$

Solving for $\xi_{x}$ and $\xi_{y}$ gives the equivalent system

$$
\begin{aligned}
& \xi_{x}=-b \eta_{x}-c \eta_{y} \\
& \xi_{y}=a \eta_{x}+b \eta_{y} .
\end{aligned}
$$

Because of the hypotheses on $a, b, c$, we can invoke a known result which says that there is a global homeomorphism $(x, y) \rightarrow(\xi, \eta)$ from $G$ onto $G^{\prime}$ such that $\xi$ and $\eta$ satisfy (18) and (19), and the Jacobian $\xi_{x} \eta_{y}-\xi_{y} \eta_{x}$ does not vanish on $G$. (See [2, p. 160], and for a more general result see [1].) There is no restriction on the domain $G$. Functions satisfying (18), (19) are necessarily in the class $H_{2}(G)$ [9, Theorem 2.4, p. 87], and in particular are $C^{2}$ functions on $G$. It follows from (4), (5), (18) that (14) becomes

$$
a^{\prime}\left(w_{\xi \xi}+w_{\eta \eta}\right)+(L \xi) w_{\xi}+(L \eta) w_{\eta}=0
$$

in the $(\xi, \eta)$ variables (cf. [2], p. 159).

THEOREM 8. There is a square-conjugate pair of functions $u, v$ for the regular elliptic operator $L$ if and only if

$$
d=a_{x}+b_{y} ; \quad e=b_{x}+c_{y}
$$

If (21) holds, and $\xi, \eta$ are a change of variables satisfying (18), (19), then (20) is Laplace's equation, and $\xi, \eta$ are square-conjugates for $L$. Functions $u, v$ are square-conjugates for $L$ if and only if $u+i v$ or $u-i v$ is an analytic function of $\xi+$ in in $G^{\prime}$.

Note. The equations (21) are just the conditions that $L$ be selfadjoint:

$$
L w=\left(a w_{x}+b w_{y}\right)_{x}+\left(b w_{x}+c w_{y}\right)_{y}
$$


Without the assumption that (14) is normalized $\left(a c-b^{2}=1\right)$, (21) becomes

$$
K d=(K a)_{x}+(K b)_{y}, \quad K e=(K b)_{x}+(K c)_{y}
$$

where $K=\left(a c-b^{2}\right)^{-1 / 2}$.

Proof. Assume that $u, v$ are square-conjugates for $L$, so that $u, v$, $u v$, and $u^{2}-v^{2}$ all satisfy (20) when considered as functions of $\xi$ and $\eta$ in $G^{\prime}$. Because $a c-b^{2}>0$, the coefficient $a^{\prime}$ of (20) (cf. (5)) only vanishes when $\xi_{x}=\xi_{y}=0$. Hence $a^{\prime} \neq 0$ on $G$ since $\xi_{x} \eta_{y}-\xi_{y} \eta_{x} \neq 0$. By Theorem 5, $L \xi=L \eta=0$ and (20) is Laplace's equation. Since $\xi$ and $\eta$ are obviously square-conjugates for Laplace's equation $w_{\xi \xi}+w_{\eta \eta}=0, \xi$ and $\eta$ are square-conjugates for $L$. We set $\eta_{x y}=\eta_{y x}$ in (18) and get

$$
\left(a \xi_{x}+b \xi_{y}\right)_{x}+\left(b \xi_{x}+c \xi_{y}\right)_{y}=0 .
$$

Subtracting $L \xi=0$ from (24) gives

$$
\left(a_{x}+b_{y}-d\right) \xi_{x}+\left(b_{x}+c_{y}-e\right) \xi_{y}=0 \text {. }
$$

Similarly, from (19) and $L \eta=0$ we get

$$
\left(a_{x}+b_{y}-d\right) \eta_{x}+\left(b_{x}+c_{y}-e\right) \eta_{y}=0 .
$$

Since the Jacobian $\xi_{x} \eta_{y}-\xi_{y} \eta_{x} \neq 0$, we conclude that $d=a_{x}+b_{y}$ and $e=b_{x}+c_{y}$.

Now assume that (21) holds; i.e., that $L$ is the self-adjoint operator (22). It follows immediately from (18) and (19) that $L \xi=L \eta=0$. Hence (20) is Laplace's equation and $\xi, \eta$ are square-conjugates for $L$.

The square-conjugate pairs $u, v$ for $L$ in $G$ correspond to the square-conjugate pairs for Laplace's equation in $G^{\prime}$. Hence by Theorem 5 the square-conjugate pairs $u, v$ for $L$ coincide with the analytic functions $u+i v$ of $\xi+i \eta$ in $G^{\prime}$.

Corollary 1. If $A$ is an algebra of complex $C^{2}$ functions on $G$ such that $L f=0$ for some regular elliptic operator and all $f \in A$, then there is a change of variables $\zeta=\xi+$ in on $G$ onto $G^{\prime}$ such that $f \circ \zeta^{-1}$ is analytic on $G^{\prime}$ for all $f \in A$.

Proof. As in Theorem 6, $A$ or $\bar{A}$ consists of analytic functions of $\xi+i \eta$. If $\bar{f}$ is an analytic function of $\xi+i \eta, f$ is an analytic function of $\xi-i \eta$ 
We know that if $(\xi, \eta)$ is a change of variables on $G$ and $\xi, \eta$ satisfy the Beltrami system (18), then $\xi$ and $\eta$ are square-conjugates for $L$. We show next that the Beltrami equations characterize square-conjugacy in general; i.e., without assuming the mapping $(x, y) \rightarrow(\xi, \eta)$ is one-toone. In fact, the Beltrami systems are simply the Cauchy-Riemann equations after a change of variable.

We consider the following two Beltrami systems, which are the same as (18) and its negative. We continue to assume that $a, b, c \in H_{1}(G)$.

$$
\begin{aligned}
& v_{x}=b u_{x}+c u_{y} \\
& v_{y}=-a u_{x}-b u_{y} \\
& v_{x}=-b u_{x}-c u_{y} \\
& v_{y}=a u_{x}+b u_{y} .
\end{aligned}
$$

LEMMA 9. If $(x, y) \rightarrow(\xi, \eta)$ is a change of variables on $G$ onto $G^{\prime}$ such that $\xi$ and $\eta$ satisfy (18), (19), then (27) is equivalent to $u_{\xi}=v_{\eta}$, $u_{\eta}=-v_{\xi}$, and (28) is equivalent to $u_{\xi}=-v_{\eta}, u_{\eta}=v_{\xi}$.

Proof. We write the first equation of (27) in terms of $\xi$ and $\eta$, using (18) and (19):

$$
\begin{aligned}
v_{\xi} \xi_{x}+v_{\eta} \eta_{x} & =b\left(u_{\xi} \xi_{x}+u_{\eta} \eta_{x}\right)+c\left(u_{\xi} \xi_{y}+u_{\eta} \eta_{y}\right) \\
& =u_{\xi}\left(b \xi_{x}+c \xi_{y}\right)+u_{\eta}\left(b \eta_{x}+c \eta_{y}\right) \\
& =u_{\xi} \eta_{x}-u_{\eta} \xi_{x}
\end{aligned}
$$

In the same way, the second equation in (27) yields

$$
v_{\xi} \xi_{y}+v_{\eta} \eta_{y}=u_{\xi} \eta_{y}-u_{\eta} \xi_{y}
$$

Hence we have the following system representing (27) in the $(\xi, \eta)$ variables:

$$
\begin{aligned}
& v_{\xi} \xi_{x}+v_{\eta} \eta_{x}=u_{\xi} \eta_{x}-u_{\eta} \xi_{x} \\
& v_{\xi} \xi_{y}+v_{\eta} \eta_{y}=u_{\xi} \eta_{y}-u_{\eta} \xi_{y}
\end{aligned}
$$

Since $\xi_{x} \eta_{y}-\xi_{y} \eta_{x} \neq 0$, we can solve for $v_{\xi}$ and $v_{\eta}$, and we get

$$
v_{\xi}=-u_{\eta}, \quad v_{\eta}=u_{\xi}
$$


Of course (30) is equivalent to (29) and (27), and similarly (28) is equivalent to

$$
v_{\xi}=u_{\eta}, \quad v_{\eta}=-u_{\xi} .
$$

THEOREM 10. If $L$ is a self-adjoint regular elliptic operator, and $u, v$ are $C^{2}$ functions on $G$, then the following are equivalent:

(a) $u, v$ are square-conjugates for $L$ in $G$

(b) $u, v$ satisfy one of the Beltrami systems (27), (28) throughout $G$

(c) $u, v$ satisfy (15), (16) in $G$.

Proof. Let $\xi$ and $\eta$ be a change of variables on $G$ onto $G^{\prime}$ such that (18) and (19) hold. Then by Theorem $8, L w=0$ becomes Laplace's equation in the $(\xi, \eta)$ variables.

Assume (a) holds. Then $u$ and $v$ are square-conjugates for Laplace's equation in $G^{\prime}$. Hence $u$ and $v$ are conjugate harmonic functions of $\xi$ and $\eta$; i.e., (30) or (31) holds, and therefore (27) or (28) holds.

To show that (b) implies (a), we assume that $u$ and $v$ satisfy (27) or (28), and hence that $u$ and $v$ are conjugate harmonic functions of $\xi$ and $\eta$ in $G^{\prime}$. Hence $u$ and $v$ are square-conjugates for Laplace's equation in $G^{\prime}$, and therefore square-conjugates for $L$ in $G$.

We have already shown (Lemma 7) that (a) implies (c), so assume (c) holds. Let $f=u+i v$, so that (17) holds:

$$
a f_{x}^{2}+2 b f_{x} f_{y}+c f_{y}^{2}=0 .
$$

Substituting $f_{x}=f_{\xi} \xi_{x}+f_{\eta} \eta_{x}, f_{y}=f_{\xi} \xi_{y}+f_{\eta} \eta_{y}$ we get

$$
f_{\xi}^{2}\left(a \xi_{x}^{2}+2 b \xi_{x} \xi_{y}+c \xi_{y}^{2}\right)+f_{\eta}^{2}\left(a \eta_{x}^{2}+2 b \eta_{x} \eta_{y}+c \eta_{y}^{2}\right)=0 .
$$

Here we used the fact that

$$
a \xi_{x} \eta_{x}+b \xi_{x} \eta_{y}+b \xi_{y} \eta_{x}+c \xi_{y} \eta_{y}=0
$$

which follows from (18). The coefficients of $f_{\xi}^{2}$ and $f_{\eta}^{2}$ are equal and nonzero. Hence $f_{\xi}^{2}+f_{\eta}^{2}=0$, which is equivalent to

$$
\begin{aligned}
& u_{\xi} v_{\xi}+u_{\eta} v_{\eta}=0 \\
& u_{\xi}^{2}+u_{\eta}^{2}=v_{\xi}^{2}+v_{\eta}^{2} .
\end{aligned}
$$

By Theorem $4, u$ and $v$ are conjugate harmonic functions on $G^{\prime}$, and hence square-conjugates for $L$. 
COROLlary 1. If $u, v$ is any square-conjugate pair for $L$, then $J=u_{x} v_{y}-u_{y} v_{x}$ is nonzero on any open subset of $G$ on which $f=u+i v$ is one-to-one. The zeros of $J$ and $f_{x}$ and $f_{y}$ are isolated.

Note. This result is proved for solutions of Beltrami systems in [9, p. 91]. We include a brief proof here for the reader's convenience.

Proof. To be specific, assume $u, v$ satisfy the Beltrami system (27). Let $\xi, \eta$ be new variables such that $u+i v$ is an analytic function of $\xi+i \eta$, with $u_{\xi}=v_{\eta}$ and $u_{\eta}=-v_{\xi}$. Then

$$
\begin{aligned}
J & =\frac{\partial(u, v)}{\partial(\xi, \eta)} \frac{\partial(\xi, \eta)}{\partial(x, y)} \\
& =\left(u_{\xi} v_{\eta}-u_{\eta} v_{\xi}\right)\left(\xi_{x} \eta_{y}-\xi_{y} \eta_{x}\right) \\
& =\left(u_{\xi}^{2}+v_{\xi}^{2}\right)\left(\xi_{x} \eta_{y}-\xi_{y} \eta_{x}\right) \\
& =\left|f^{\prime}\right|^{2}\left(\xi_{x} \eta_{y}-\xi_{y} \eta_{x}\right) .
\end{aligned}
$$

The zeros of $f^{\prime}$ are isolated, and $f^{\prime}$ is not zero on any open set on which $f$ is one-to-one.

Corollary 2. If $A$ is an algebra of complex $C^{2}$ functions on $G$ which satisfy (14), where $L$ is a regular elliptic operator, then $L$ is self-adjoint and (27) or (28) holds for every $u+i v$ in $A$.

Proof. Either every $f$ is an analytic function of $\xi+i \eta$ or every $\bar{f}$ is an analytic function of $\xi+i \eta$ by Theorem 6 .

Next we characterize those pairs $u, v$ of $\mathrm{H}_{2}(G)$ functions which are square-conjugates for some regular elliptic operator $L$. We show there is at most one such $L$ for any pair $u, v$. We also give a simple geometric condition on $u$ and $v$ which characterizes the fact that $u+i v$ is an analytic function composed with a homeomorphism.

THEOREM 11. Let $f$ be a nonconstant function in $H_{2}(G)$. If $f$ satisfies

$$
L(f)=L\left(f^{2}\right)=0
$$

for some regular elliptic operator $L$ on $G$, then $f$ satisfies

$$
f_{x}=\lambda f_{y}
$$


for some complex function $\lambda \in H_{1}(G)$ with $\operatorname{Im} \lambda \neq 0$, and $\lambda$ is determined up to complex conjugation by the coefficients of $L$.

Conversely, if $f$ satisfies (35) in $G$ for a complex function $\lambda$ in $H_{1}(G)$, with $\operatorname{Im} \lambda \neq 0$, then there is a unique regular elliptic operator $L$ such that $L(f)=L\left(f^{2}\right)=0$.

Proof. If $L(f)=L\left(f^{2}\right)=0$, then by Lemma 7 we have

$$
a f_{x}^{2}+2 b f_{x} f_{y}+c f_{y}^{2}=0 .
$$

The zeros of $f_{x}$ and $f_{y}$ are isolated by Corollary 1 of Theorem 10, so the quadratic equation (36) gives

$$
f_{x}=(-b / a \pm i / a) f_{y}
$$

with $\lambda=-b / a \pm i / a$ uniquely determined except for the sign of $\operatorname{Im} \lambda$. Since $a$ never vanishes and $f_{y}$ vanishes at most at isolated points, the sign of $\operatorname{Im} \lambda$ is constant in $G$.

Now assume that (35) holds, with $\operatorname{Im} \lambda>0$ to be specific. We let

$$
a=1 / \operatorname{Im} \lambda, \quad b=-\operatorname{Re} \lambda / \operatorname{Im} \lambda, \quad c=|\lambda|^{2} / \operatorname{Im} \lambda .
$$

Then $a c-b^{2}=1, a>0$, and $a, b, c \in H_{1}(G)$. It is easy to check that (36) holds, so $L(f)=L\left(f^{2}\right)=0$ by (c) of Theorem 10. Equation (37) shows that $L$ is uniquely determined by $\lambda$, given that $a c-b^{2}=1$ and $a>0$.

COROLLARY 1. If $L$ is a regular elliptic operator and $L(f)=L\left(f^{2}\right)=$ 0 , then $L(\phi \circ f)=0$ for every $\phi$ analytic on $f(G)$.

Proof. If $g=\phi \circ f$, then $g_{x}=\left(\phi^{\prime} \circ f\right) f_{x}$ and $g_{y}=\left(\phi^{\prime} \circ f\right) f_{y}$, so $g_{x}=\lambda g_{y}$ if $f_{x}=\lambda f_{y}$.

Corollary 2. If $f \in H_{2}(G)$, and $f$ is nonconstant, there is at most one regular elliptic operator $L$ on $G$ such that $L(f)=L\left(f^{2}\right)=0$, and there is at most one $\lambda \in H_{1}(G)$ with $\operatorname{Im} \lambda \neq 0$ such that $f_{x}=\lambda f_{y}$.

Corollary 3. If $f=u+i v \in H_{2}(G)$ and $J=u_{x} v_{y}-u_{y} v_{x}$ does not vanish on $G$, then there is a unique regular elliptic operator $L$ on $G$ such that $L(f)=L\left(f^{2}\right)=0$.

Proof. If $J \neq 0$, then $f_{y} \neq 0$, and if $\lambda=f_{x} / f_{y}$, then $\operatorname{Im} \lambda=$ $-J /\left|f_{y}\right|^{2} \neq 0$. 
The Cauchy-Riemann equations can be written $f_{x}=-i f_{y}$, where $f=u+i v$. The following can therefore be considered a generalization of the Cauchy-Riemann characterization of analyticity:

THEOREM 12. If $f \in H_{2}(G)$ and $f_{x}=\lambda f_{y}$ for some $\lambda \in H_{1}(G)$ with $\operatorname{Im} \lambda \neq 0$, then $f=\phi \circ \zeta$ where $\zeta \in H_{2}(G)$ is a homeomorphism of $G$, and $\phi$ is analytic on $\zeta(G)$.

Proof. We know that $f$ or $\bar{f}$ is an analytic function of $\zeta=\xi+i \eta$, where $\xi+i \eta$ is a homeomorphism and $\xi, \eta$ satisfy (18), and $\zeta \in H_{2}(G)$ by [9, Theorem 2.4, p. 87]. If $\bar{f}$ is an analytic function of $\zeta$, then $f$ is an analytic function of $\bar{\zeta}$.

A geometric interpretation of the condition $f_{x}=\lambda f_{y}, \operatorname{Im} \lambda \neq 0$, can be given as follows. If the complex quantities $f_{x}$ and $f_{y}$ are considered as vectors in two-space, the condition implies that these vectors are nonparallel whenever they are nonzero. But $f_{x}$ and $f_{y}$ are nonzero and nonparallel at the same time that $\nabla u$ and $\nabla v$ are nonzero and nonparallel, as can be seen by considering the $2 \times 2$ matrix whose rows are $\nabla u$ and $\nabla v$ and whose columns are $f_{x}$ and $f_{y}$. Thus for the case when $\nabla u$ and $\nabla v$ do not vanish, the hypothesis of Theorem 12 is simply that $\nabla u$ and $\nabla v$ are nonparallel and $u, v \in H_{2}(G)$.

5. Algebras satisfying a maximum principle. In this section we use the results of $\S 4$ to describe certain algebras which satisfy a maximum principle. These results extend those of Rudin [7].

Definition. We will say that an algebra of continuous complex functions on $G$ satisfies the maximum principle on $G$ if for every compact subset $K$ of $G$ and every $f \in A, \quad \max \{|f(z)|: z \in K\}=$ $\max \{|f(z)|: z \in \partial K\}$.

THEOREM 13. Let $A$ be an algebra of complex functions in $H_{2}(G)$ which satisfies the maximum principle. If

$$
L(f)=L\left(f^{2}\right)=0
$$

for some nonconstant $f \in A$ and some regular elliptic operator $L$, then (39) holds for all $f \in A$. If

$$
f_{x}=\lambda f_{y}
$$

for some nonconstant $f \in A$ and some $\lambda \in H_{1}(G)$ with $\operatorname{Im} \lambda \neq 0$, then every function in A satisfies (40). 
Proof. Let $f$ be a nonconstant function in $A$ which satisfies (39). By Theorem 8 , there is a change of variables $(x, y) \rightarrow(\xi, \eta)$ such that (39) becomes Laplace's equation, and $f$ is an analytic function of $\zeta=\xi+i \eta$. (Replace $\zeta$ with $\bar{\zeta}$ if $\bar{f}$ is analytic.) Rudin has shown [7, Theorem 2] that, in an algebra satisfying the maximum principle, if one nonconstant function is analytic then every function is analytic. Thus every $g \in A$ is an analytic function of $\zeta$. Again using Theorem 8, we conclude that every $g \in A$ satisfies (39). If $f$ is nonconstant and satisfies (40), then by Theorem $12, f=\phi \circ \zeta$ where $\zeta \in H_{2}(G)$ is a homeomorphism of $G$, and $\phi$ is analytic on $\zeta(G)$. Since $f_{x}-\lambda f_{y}=0=$ $\left(\phi^{\prime} \circ \zeta\right)\left(\zeta_{x}-\lambda \zeta_{y}\right)$ and the zeros of $\phi^{\prime}$ are isolated, $\zeta_{x}=\lambda \zeta_{y}$. Again by Rudin's result, every $g \in A$ is an analytic function of $\zeta$. It follows that $g_{x}=\lambda g_{y}$.

We next give a local criterion that an algebra satisfying the maximum principle consists of analytic functions after a change of variables.

THeOREM 14. Let $A$ be an algebra of functions in $H_{2}(G)$ which satisfies the maximum principle. Suppose that at each point $z$ in $G$ there exists an open sphere $S_{z} \subset G$ centered at $z$ and a function $\lambda_{z} \in H_{1}\left(S_{z}\right)$ with $\operatorname{Im} \lambda_{z} \neq 0$, and a function $f_{z}$ in $A$, nonconstant in $S_{z}$, such that

$$
\frac{\partial f_{z}}{\partial x}=\lambda_{z} \frac{\partial f_{z}}{\partial y}
$$

in $S_{z}$. Then there is a change of variables $\zeta=\xi+$ in from $G$ onto $G^{\prime}$ such that $\zeta \in H_{2}(G)$ and $f \circ \zeta^{-1}$ is analytic on $G^{\prime}$ for all $f \in A$.

(Note that if at every point $z \in G$ the algebra contains a function with non-vanishing Jacobian at $z$ then the conditions of the theorem are satisfied, by Corollary 3 to Theorem 11.)

Proof. It is sufficient to show that there exists $\lambda \in H_{1}(G), \operatorname{Im} \lambda \neq 0$, such that $f_{x}=\lambda f_{y}$ for all $f \in A$. The result will then follow from Theorem 11 and Corollary 1 to Theorem 8. By applying Theorem 13 to each domain $S_{z}$, we conclude that $f_{x}=\lambda_{z} f_{y}$ in $S_{z}$ for every $f \in A$, $z \in G$. We will show that if two spheres $S_{z_{1}}$ and $S_{z_{2}}$ overlap, then $\lambda_{z_{1}}=\lambda_{z_{2}}$ in the intersection, and hence $\lambda$ is defined globally. But in $S_{z_{1}} \cap S_{z_{2}}$, the function $f_{z_{1}}$ satisfies (41) and the corresponding equation with $\lambda_{z_{1}}$ replaced by $\lambda_{z_{2}}$. Since the zeros of $\partial f_{z} / \partial y$ are isolated in $S_{z}$ (Corollary 1 to Theorem 10), we must have $\lambda_{z_{1}}=\lambda_{z_{2}}$ in the intersection. 


\section{REFERENCES}

1. Lipman Bers, Univalent solutions of linear elliptic systems, Comm. on Pure and Appl. Math., VI (1953), 513-526.

2. Richard Courant and David Hilbert, Methods of Mathematical Physics, Interscience, New York, 2 (1965).

3. V. K. Dzyadyk, Geometrical definition of analytic functions (Russian), Uspehi Mat. Nauk, 15 (1960), No. 1 (91), 191-194.

4. W. P. Novinger, Real parts of uniform algebras on the circle, Pacific J. Math., 57 (1975), 259-264.

5. J. M. F. O'Connell, Real parts of uniform algebras, Pacific J. Math., 46 (1973), 235-247.

6. I. G. Petrowskii, Partial Differential Equations, W. B. Saunders Co., Philadelphia, 1967.

7. Walter Rudin, Analyticity and the maximum modulus principle, Duke Math. J., 20 (1953), 449-457.

8. - Real and Complex Analysis, second edition, McGraw-Hill, New York, 1974.

9. I. N. Vekua, Generalized Analytic Functions, Pergamon Press, London, 1962.

Received March 24, 1977 and in revised form May 16, 1977. The second author was supported in part by grant MCS76-07180 of the National Science Foundation.

UNIVERSITY OF HAWAII

HoNOLULU, HI 96822 


\section{PACIFIC JOURNAL OF MATHEMATICS EDITORS}

ICHARD ARENS (Managing Editor)

niversity of California

os Angeles, CA 90024

\section{. A. BEAUmont}

niversity of Washington

sattle, WA 98105

. C. MOORE

niversity of California

erkeley, CA 94720
J. DUGUNDJI

Department of Mathematics

University of Southern California

Los Angeles, CA 90007

R. FINN AND J. MiLgRAM

Stanford University

Stanford, CA 94305

\section{ASSOCIATE EDITORS}
F. BECKENBACH
B. H. NEUMANN
F. WOLF
K. YosHidA

\section{SUPPORTING INSTITUTIONS}

NIVERSITY OF BRITISH COLUMBIA

UNIVERSITY OF SOUTHERN CALIFORNIA

ALIFORNIA INSTITUTE OF TECHNOLOGY STANFORD UNIVERSITY

NIVERSITY OF CALIFORNIA

ONTANA STATE UNIVERSITY

UNIVERSITY OF HAWAII

NIVERSITY OF NEVADA

UNIVERSITY OF TOKYO

EW MEXICO STATE UNIVERSITY

UNIVERSITY OF UTAH

REGON STATE UNIVERSITY

NIVERSITY OF OREGON

WASHINGTON STATE UNIVERSITY

UNIVERSITY OF WASHINGTON

SAKA UNIVERSITY

AMERICAN MATHEMATICAL SOCIETY

The Supporting Institutions listed above contribute to the cost of publication of this Journal, but they a t owners or publishers and have no responsibility for its contents or policies.

Mathematical papers intended for publication in the Pacific Journal of Mathematics should be in typ Irm or offset-reproduced (not dittoed), double spaced with large margins. Underline Greek letters in re ierman in green, and script in blue. The first paragraph or two must be capable of being used separately as 'nopsis of the entire paper. Items of the bibliography should not be cited there unless absolutely necessary, hich case they must be identified by author and Journal, rather than by item number. Manuscripts, uplicate, may be sent to any one of the four editors. Please classify according to the scheme of Math. Revieu Idex to Vol. 39. All other communications should be addressed to the managing editor, or Elaine Bart Iniversity of California, Los Angeles, California, 90024.

100 reprints are provided free for each article, only if page charges have been substantially pai dditional copies may be obtained at cost in multiples of 50 .

The Pacific Journal of Mathematics is issued monthly as of January 1966. Regular subscription rate: \$72. year (6 Vols., 12 issues). Special rate: $\$ 36.00$ a year to individual members of supporting institutions.

Subscriptions, orders for numbers issued in the last three calendar years, and changes of address should ent to Pacific Journal of Mathematics, 103 Highland Boulevard, Berkeley, California, 94708.

UBLISHED BY PACIFIC JOURNAL OF MATHEMATICS, A NON-PROFIT CORPORATION

Printed at Jerusalem Academic Press, POB 2390, Jerusalem, Israel.

Copyright (C) 1978 Pacific Journal of Mathematics

All Rights Reserved 


\section{Pacific Journal of Mathematics}

\section{Vol. 75, No. $1 \quad$ September, 1978}

Mieczyslaw Altman, General solvability theorems

Denise Amar and Eric Amar, Sur les suites d'interpolation en plusieurs variables ..........................................

Herbert Stanley Bear, Jr. and Gerald Norman Hile, Algebras which satisfy a second order linear partial differential equation ..................

Marilyn Breen, Sets in $R^{d}$ having $(d-2)$-dimensional kernels ............

Gavin Brown and William Moran, Analytic discs in the maximal ideal space

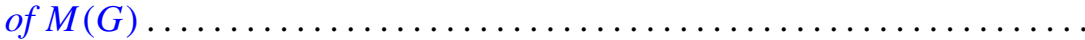

Ronald P. Brown, Quadratic forms with prescribed Stiefel-Whitney

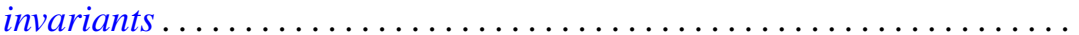

Gulbank D. Chakerian and H. Groemer, On coverings of Euclidean space by convex sets

S. Feigelstock and Z. Schlussel, Principal ideal and Noetherian groups.....

Ralph S. Freese and James Bryant Nation, Projective lattices ............

Harry Gingold, Uniqueness of linear boundary value problems for

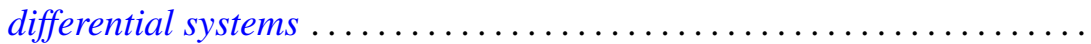

John R. Hedstrom and Evan Green Houston, Jr., Pseudo-valuation domains. . .

William Josephson, Coallocation between lattices with applications to measure extensions

M. Koskela, A characterization of non-negative matrix operators on $l^{p}$ to $l^{q}$ with $\infty>p \geq q>1$

Kurt Kreith and Charles Andrew Swanson, Conjugate points for nonlinear differential equations...........................

Shoji Kyuno, On prime gamma rings ........................ 185

Alois Andreas Lechicki, On bounded and subcontinuous multifunctions ..

Roberto Longo, A simple proof of the existence of modular automorphisms in approximately finite-dimensional von Neumann algebras ...

Kenneth Millett, Obstructions to pseudoisotopy implying isotopy for

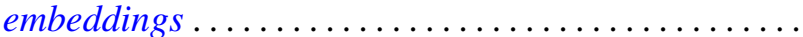

William F. Moss and John Piepenbrink, Positive solutions of elliptic equations. .

Mitsuru Nakai and Leo Sario, Duffin's function and Hadamard's

conjecture

Mohan S. Putcha, Word equations in some geometric semigroups ...

Walter Rudin, Peak-interpolation sets of class $C^{1} \ldots \ldots \ldots$

Elias Saab, On the Radon-Nikodým property in a class of locally convex

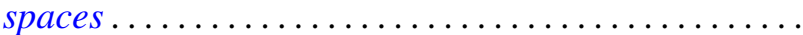

